\title{
Basic Superposition is Complete
}

\author{
Robert Nieuwenhuis and Albert Rubio \\ Universidad Politécnica de Cataluña \\ Dept. Lenguajes y Sistemas Informáticos \\ Pau Gargallo 5, E-08028 Barcelona, Spain \\ E-mail: robertoelsi.upc.es rubio@lsi.upc.es
}

\begin{abstract}
We define equality constrained equations and clauses and use them to prove the completeness of what we have called basic superposition: a restricted form of superposition in which only the subterms not created in previous inferences is superposed upon. We first apply our results to the equational case and define an unfailing Knuth-Bendix completion procedure that uses basic superposition as inference rule. Second, we extend the techniques to completion of full first-order clauses with equality. Moreover, we prove the refutational completeness of a new simple inference system.
\end{abstract}

\section{Introduction}

Reasoning about equality has many applications in computer science, including automated theorem proving, logic and equational programming, symbolic algebraic computation, and program specification and verification. Knuth-Bendix-like completion techniques [KB 70, Rus 87, HR 89, BDP 89, BG 91, NO 91] are one of the most successful approaches for dealing with equality. Completion procedures can be seen as refutationally complete processes that moreover transform sets of axioms in such a way that, by using the final complete set, efficient normal form proof strategies become complete (e.g. rewrite proofs or linear proofs). Completion is normally based on a form of paramodulation with strong ordering restrictions, called superposition.

In this paper we develop a notion of equality constraints and use it to prove the completeness of basic superposition. This result has important consequences for KnuthBendix completion of equations and other first-order clauses with equality, and has been searched for since the completeness of basic narrowing was proved in [Hul 80]. Roughly speaking, the inference rule of basic superposition is the restriction of normal superposition in which the only inferences that have to be computed are the ones at subterms that have not been created in previous inference steps. Consider for example the inference by (equational) superposition

$$
\frac{f(g(a)) \simeq a \quad h(f(x)) \simeq h(x)}{h(a) \simeq h(g(a))}
$$

obtained by unifying in $h(f(x))$ the subterm $f(x)$ with $f(g(a))$. Its conclusion is an instance with the unifier $\{x \mapsto g(a)\}$ of the equation $h(a) \simeq h(x)$. Therefore, no further basic superposition steps have to be applied to subterms of $g(a)$ in this conclusion, whereas in normal superposition all subterms of $h(g(a))$ must be considered. 
In this paper we will describe such situations by means of equations with equality constraints. In the example above the conclusion would be $h(a) \simeq h(x) \llbracket x=g(a) \rrbracket$, i.e. the instantiations caused by inference steps are kept in the constraints. Normal superposition can then be used for the non-variable subterms of the equation part (in the example, $h(a) \simeq h(x)$ ). The inference rule of (here for simplicity equational) basic superposition can then be expressed as

$$
\frac{s \simeq s^{\prime} \llbracket T^{\prime} \rrbracket \quad t \simeq t^{\prime} \llbracket T \rrbracket}{\left.t\left[s^{\prime}\right]_{u} \simeq t^{\prime} \llbracket T \wedge T^{\prime} \wedge t\right|_{u}=s \rrbracket} \quad \text { where }\left.t\right|_{u} \notin \mathcal{V} \text { ars }(t)
$$

if moreover the usual ordering restrictions for superposition are fulfilled. As we can see, equality constraints provide a simple and elegant representation for this inference rule. Information from the meta-level, in this case the accumulated unifiers, is kept in the constraints and used later on. Of course, other notations and practical implementations for basic superposition are possible, such as pairs clause-substitution or clauses in which "forbidden" subterms are marked somehow.

Obviously, basic superposition is a considerable improvement over normal superposition as defined in e.g. [BG 91], allowing to importantly reduce the search space, and to obtain complete systems in more cases. One of the reasons is that by normal superposition many superfluous consequences are generated. Sometimes one can try to eliminate these consequences (e.g. in the equational case some -but not all-redundant critical pairs are joinable), but this is not always possible (almost never in the nonequational cases), and very expensive in general. By basic superposition, many of these superfluous consequences are simply not created.

This paper is structured as follows. After the basic definitions of section 2, in the third section we apply our techniques to the particular case of equational logic and define a new unfailing Knuth-Bendix completion procedure that uses basic superposition as inference rule. In section 4 we extend the results to the case of Horn-clauses with equality and further to full first-order clauses with equality. Our style of proof is based on the model construction techniques and redundancy notions defined by Bachmair and Ganzinger in [BG 91]. We prove the refutational completeness of a basic superpositionbased inference system, which moreover uses a simple new factoring rule. Section 5 is on further work.

Related work (simultaneously and independently developed) on similar "basic" restrictions, but for paramodulation, has recently been presented by W. Snyder and C. Lynch at the UNIF-91 workshop in Barbizon, France. Their method is less useful for Knuth-Bendix completion, since it needs paramodulation on right hand sides, and gives no simplification and deletion mechanisms for redundant equations and clauses. Their proof methods are completely different from ours and more complex. We have also learned that L. Bachmair, H. Ganzinger, C. Lynch and W. Snyder have very recently further developed the previous method obtaining basic superposition calculi more similar to ours. 


\section{Basic notions and terminology}

We adopt the standard notations and definitions for term rewriting given in [DJ 90, 91].

Furthermore, by equality constraints we mean conjunctions of equalities of terms $t=t^{\prime}$, where $=$ denotes syntactic equality of the terms $t$ and $t^{\prime}$. An equality constraint $T$ of the form $t_{1}=t_{1}^{\prime} \wedge \ldots \wedge t_{n}=t_{n}^{\prime}$ is satisfiable iff there exists a (most general) unifier $\sigma$ of $T$, i.e. $\sigma$ simultaneously unifies every $t_{i}$ with $t_{i}^{\prime}$ for $i=1 \ldots n$. Every unifier $\theta$ of $T$ is called a solution of $T$, and then we say that $T \theta$ is (equivalent to) true, denoted $T \theta \equiv$ true.

By an equation we mean a multiset $\{s, t\}$, denoted by $s \simeq t$ (or equivalently by $t \simeq s$, where $s$ and $t$ are terms in $\mathcal{T}(\mathcal{F}, \mathcal{X})$. A first-order clause $\Gamma \rightarrow \Delta$ is a pair of (finite) multisets of equations $\Gamma$ and $\Delta$, called respectively the antecedent and the succedent of the clause.

An equality constrained clause is a pair $(C, T)$, denoted $C \llbracket T \rrbracket$, where $C$ is a clause and $T$ is an equality constraint. Such a pair can be seen as a shorthand for the set of ground instances of $C \llbracket T \rrbracket$ : those ground clauses $C \sigma$ such that $T \sigma$ is true. We will suppose distinct equality constrained clauses not to share variables.

Like in [BG 91], here we consider interpretations that are congruences on ground terms. An interpretation $I$ satisfies a ground clause $\Gamma \rightarrow \Delta$, denoted by $I \mid \Gamma \rightarrow \Delta$, if $I \nsupseteq \Gamma$ or else $I \cap \Delta \neq \emptyset$. An interpretation $I$ satisfies (is a model of) $C \llbracket T \rrbracket$, denoted $I \models C \llbracket T \rrbracket$, if it satisfies every ground instance of $C \llbracket T \rrbracket$, i.e. clauses with unsatisfiable constraints are tautologies. The empty clause (with a satisfiable constraint!) is satisfied by no interpretation. I satisfies a set of clauses $S$, denoted by $I \models S$, if it satisfies every clause in $S$. A clause $C$ can be deduced from a set of clauses $S$ (denoted by $S \models C$ ), if $C$ is satisfied by every model of $S$.

For dealing with non-equality predicates, we express atoms $A$ by equations $A \simeq$ true where true is a special symbol, i.e. we treat atoms as terms. Here $\succ$ denotes a total simplification ordering on ground terms, where the special symbol true is the smallest symbol. We use $\succ_{m u l}\left(\succ_{m u l^{n}}\right)$ to denote its $(n$-fold) multiset extension.

We use the definitions of [DJ 91] for rewriting-related notions like normal form, confluence, convergence, reducibility, etc. We denote ground rewrite rules (ground equations $t \simeq t^{\prime}$ with $t \succ t^{\prime}$ ) by $t \Rightarrow t^{\prime}$. The congruence generated by a set of ground rewrite rules $R$ (which is an interpretation) will be denoted by $R^{*}$. 


\section{Basic superposition in the equational case}

In this section we deal with clauses of the form $\rightarrow s \simeq t$, denoted here by $s \simeq t$.

Definition 1: The inference rule of equational basic superposition is defined as follows:

$$
\frac{s \simeq s^{\prime} \llbracket T^{\prime} \rrbracket \quad t \simeq t^{\prime} \llbracket T \rrbracket}{\left.t\left[s^{\prime}\right]_{u} \simeq t^{\prime} \llbracket T \wedge T^{\prime} \wedge t\right|_{u}=s \rrbracket} \quad \text { where }\left.t\right|_{u} \notin \mathcal{V} \text { ars }(t)
$$

if, for some ground substitution $\sigma,\left[\left.T \wedge T^{\prime} \wedge t\right|_{u}=s \rrbracket \sigma\right.$ is true, $s \sigma \succ s^{\prime} \sigma$ and $t \sigma \succ t^{\prime} \sigma$.

As said, constraint solving for equality constraints is just unification (in practice, every satisfiable constraint $T$ can be kept in a simplest form, which is its most general unifier, and non-relevant variables can be eliminated, although here we will not go into these details).

The difficulty with basic superposition (and with all forms of deduction with constrained formulae) is that lifting lemmas like the critical pair lemma [KB 70] do not hold:

Example 2: No inference by basic superposition can be made between the two equations $a \simeq b$ and $f(x) \simeq b \llbracket x=a \rrbracket$, where $a \succ b$. Now the term $f(a)$ rewrites into $f(b)$ by the first equation, and into $b$ by the second one, but there is no term $t$ such that $f(b)$ and $b$ are both reducible to $t$, i.e. the critical pair lemma does not hold when considering only critical pairs by basic superposition.

Another conclusion that we can draw from this example is that basic superposition is not complete as inference rule for equational Knuth-Bendix completion when starting from an arbitrary set of equations with equality constraints: there is no rewrite proof at all for the consequence $f(b) \simeq b$, although the set of equations is closed under basic superposition.

Therefore, here we will suppose that the initial set of axioms contains only clauses without constraints*, i.e. clauses of the form $C \llbracket T \rrbracket$ where $T$ is an empty (or trivially true) constraint, sometimes written $C \llbracket t r u e \rrbracket$.

For simplicity, we will first study basic superposition without simplification. It is proved that the closure under basic superposition of an initial set of equations without constraints is ground confluent. We do this by first defining a (canonical) set of ground rewrite rules $R_{E}$ generated from a set $E$ of equations, by selecting ground instances of equations in $E$ that fulfil certain properties (this is similar to [BG 91], but adapted to equations with equality constraints). Then we show that $R_{E}^{*} \models E$ if $E$ is closed under basic superposition, and we prove that this implies that $E$ is ground confluent.

To overcome the problems of the non-existence of a critical pair lemma, we will sometimes consider only ground instances of equations with irreducible substitutions, defined as follows:

* In fact, this restriction can be slightly weakened 
Definition 3: A ground substitution $\sigma$ is irreducible wrt. a set of ground rewrite rules $R$ if $x \sigma$ is irreducible wrt. $R$, for every variable $x$ in the domain of $\sigma$. A normal form of $\sigma$ wrt. $R$ is a substitution $\sigma^{\prime}$ with the same domain as $\sigma$, and such that $x \sigma^{\prime}$ is a normal form wrt. $R$ of $x \sigma$, for every variable $x$ in the domain.

Definition 4: Let $s \sigma \simeq t \sigma$ be a ground instance with $s \sigma \succ t \sigma$ of an equation $s \simeq t \llbracket T \rrbracket$ in a set of equations $E$. Then $s \sigma \simeq t \sigma$ generates the rule $s \sigma \Rightarrow t \sigma$ if $s \sigma$ and $\sigma$ are irreducible wrt. the rules generated by ground instances $e \theta$ of equations in $E$ with $s \sigma \simeq t \sigma \succ_{m u l} e \theta$.

The set of rules generated by all ground instances of equations in $E$ is denoted by $R_{E}$.

Definition 5: Let $E$ be a set of constrained equations, and let $R$ be a set of ground rewrite rules. The set of ground instances of equations in $E$ with substitutions that are irreducible wrt. $R$ is denoted by $\operatorname{irred}_{R}(E)$, i.e.

$$
\operatorname{irred}_{R}(E)=\{e \sigma \mid e \llbracket T \rrbracket \in E, T \sigma \equiv \text { true, } \sigma \text { ground, } \sigma \text { irreducible wrt. } R\}
$$

Lemma 6: Let $s \simeq s^{\prime} \llbracket T \rrbracket$ be an equation in $E$ such that $s \theta \simeq s^{\prime} \theta$ generates the rule $s \theta \Rightarrow s^{\prime} \theta$ for some ground substitution $\theta$. Then $x \theta$ is irreducible wrt. $R_{E}$ for every $x$ in $\operatorname{Vars}\left(s^{\prime}\right)$.

Proof. If $s \theta \Rightarrow s^{\prime} \theta$ is generated as a rule, then $x \theta$ is irreducible wrt. the rules generated by ground instances smaller wrt. $\succ_{m u l}$ than $s \theta \simeq s^{\prime} \theta$, and also $s \theta \succ s^{\prime} \theta$. All rules generated by instances greater or equal than $s \theta \simeq s^{\prime} \theta$ have left hand sides that are strictly greater than $s^{\prime} \theta$. Therefore, none of these rules can reduce a subterm of $s^{\prime} \theta$.

Lemma 7: Let $E$ be a set of equations with equality constraints that is closed under basic superposition. Then $R_{E}^{*}=\operatorname{irred}_{R_{E}}(E)$.

Proof. We will derive a contradiction from the existence of a minimal (wrt. $\succ_{m u l}$ ) element $t \sigma \simeq t^{\prime} \sigma$ in irred $_{R_{E}}(E)$ such that $R_{E}^{*} \not \models t \sigma \simeq t^{\prime} \sigma$.

Let $t \sigma \simeq t^{\prime} \sigma$ be a ground instance of an equation $t \simeq t^{\prime} \llbracket T \rrbracket$ in $E$. We can suppose w.l.o.g. that $t \sigma \succ t^{\prime} \sigma$. Since $R_{E}^{*} \not t \sigma \simeq t^{\prime} \sigma$, the equation does not generate any rule in $R_{E}$. Therefore $t \sigma$ must be reducible by $R_{E}$, e.g. with a rule $s \theta \Rightarrow s^{\prime} \theta$ generated by an equation $s \theta \simeq s^{\prime} \theta$ smaller (wrt. $\succ_{m u l}$ ) than $t \sigma \simeq t^{\prime} \sigma$, where $s \theta \simeq s^{\prime} \theta$ is a ground instance of an equation $\left.s \simeq s^{\prime} \llbracket T^{\prime}\right]$ in $E$. Now we have $\left.t \sigma\right|_{u}=s \theta$, where $\left.t\right|_{u}$ cannot be a variable, since $\sigma$ is irreducible wrt. $R_{E}$, and therefore the following inference can be made:

$$
\frac{s \simeq s^{\prime} \llbracket T^{\prime} \rrbracket \quad t \simeq t^{\prime} \llbracket T \rrbracket}{\left.t\left[s^{\prime}\right]_{u} \simeq t^{\prime} \llbracket T \wedge T^{\prime} \wedge t\right|_{u}=s \rrbracket}
$$

Since $E$ is closed under basic superposition, its conclusion is in $E$. It has a ground instance $d$ of the form $t \sigma\left[s^{\prime} \theta\right]_{u} \simeq t^{\prime} \sigma$ such that $R_{E}^{*} \mid \neq d$ (otherwise $R_{E}^{*} \mid=t \sigma \simeq t^{\prime} \sigma$ ). Moreover, by the previous lemma and since $\sigma$ is irreducible by $R_{E}$, the instance $d$ is an instance of this conclusion with a ground substitution that is irreducible by $R_{E}$. Furthermore, we have $t \sigma \simeq t^{\prime} \sigma \succ_{m u l} d$, which altogether contradicts the minimality of $t \sigma \simeq t^{\prime} \sigma$. 
Lemma 8: Let $E_{0}$ be a set of equations without constraints, and let $E$ be the closure of $E_{0}$ under basic superposition. Then $R_{E}^{*} F E$.

Proof. First note that $E_{0} \models E$, by soundness of basic superposition. Therefore, it suffices to show that $R_{E}^{*} \models E_{0}$, i.e. $R_{E}^{*}=e \sigma$ for every ground instance $e \sigma$ of an equation $e \llbracket t r u e \rrbracket$ in $E_{0}$. Now let $\sigma^{\prime}$ be a normal form of $\sigma$ wrt. $R_{E}$. Since $E_{0} \subseteq E$, by the previous lemma it holds that $R_{E}^{*}=e \sigma^{\prime}$, because $\sigma^{\prime}$ is irreducible wrt. $R_{E}$, and $e \sigma^{\prime}$ is an existing instance of $e \llbracket$ true $\rrbracket$. From $R_{E} \cup\left\{e \sigma^{\prime}\right\} \models e \sigma$ and $R_{E}^{*} \models e \sigma^{\prime}$ it follows that $R_{E}^{*} \vDash e \sigma$.

Lemma 9: Let $E$ be a set of constrained equations such that $R_{E}^{*} \models E$. Then $E$ is ground confluent.

Proof. Let $s, s^{\prime}$ and $t$ be ground terms such that $s$ and $s^{\prime}$ are normal forms of $t$ wrt. $E$. We prove that $s$ and $s^{\prime}$ must be syntactically equal. We have $E \models s \simeq s^{\prime}$, and $R_{E}^{*} \models E$, which implies $R_{E}^{*}=s \simeq s^{\prime}$ and $R_{E} \mid=s \simeq s^{\prime}$. If $s$ and $s^{\prime}$ are normal forms wrt. $E$, then they are also normal forms wrt. $R_{E}$, because $R_{E}$ is a set of instances of equations of $E$. Moreover, by its construction, $R_{E}$ is a canonical set of ground rewrite rules because there are no overlappings between left hand sides. This implies that $s$ and $s^{\prime}$ are equal.

Theorem 10: Let $E_{0}$ be a set of equations without constraints, and let $E$ be the closure of $E_{0}$ under basic superposition. Then $E$ is ground confluent.

\subsection{Completion by basic superposition: the equational case}

Now we know that if $E$ is the closure under basic superposition of a set of equations without constraints, then $E$ is ground confluent. In this section we show that basic superposition is also the appropriate inference rule for unfailing Knuth-Bendix completion, i.e. for computing ground confluent sets in practice, even when applying the existing powerful simplification and deletion methods that can be used in normal superposition-based completion. However, at first sight there seems to be a problem with simplification:

Example 11: Consider the ordering $f \succ g \succ a \succ b$ and three initial equations:

1) $a \simeq b$

2) $f(g(x)) \simeq g(x)$

3) $f(g(a)) \simeq b$

Now a completion process including simplification could generate:

4) $g(x) \simeq b \llbracket x=a \rrbracket$

(by basic superposition of 2 and 3 )

5) $f(b) \simeq b$

(simplifying 3 by 4 )

6) $f(b) \simeq g(x) \llbracket x=a \rrbracket$

(by basic superposition of 2 and 4 )

Now the set $\{1,2,4,5,6\}$ is closed under basic superposition, i.e. this set would be the final set generated by the completion process. However, there is no rewrite proof for $g(b) \simeq b$ using instances of this set. The conclusion of this example is that, even when starting with equations without constraints, it is incorrect to apply simplification steps like the one made above, where the equation $f(g(a)) \simeq b$ is simplified into $f(b) \simeq b$ using 
the instance $g(a) \simeq b$ of $g(x) \simeq b \llbracket x=a \rrbracket$, which would be a quite natural simplification method.

However, as we will see, this problem appears only in (quite special) concrete situations, and can be solved in such a way that all intuitive simplification and deletion techniques can be allowed if sometimes certain equality constraints are slightly weakened.

Our notions of completion and redundancy are based on the ones defined in [BG 91], where an axiom is redundant if all its ground instances can be deduced from smaller instances of other axioms. Analogously, an inference is redundant if, for all its instances, the conclusion can be deduced from instances smaller than the maximal premise. These redundancy notions include, as far as we know, all correct methods that make completion procedures more efficient and terminate in more cases. Here we adapt these notions by considering only instances with substitutions that are, in some sense, irreducible.

Now we first give some definitions, which we do not pretend to be constructive. For instance, the definition of completion derivations below does not provide (yet) a way to compute them (at least not if the redundancy notions are exploited). This point will be made clear below.

Definition 12: Let $E_{0}, E_{1}, \ldots$ be a sequence of sets of constrained equations.

a) The set $E_{\infty}$ of persistent equations in $E_{0}, E_{1}, \ldots$ is defined as $\cup_{j}\left(\cap_{k \geq j} E_{k}\right)$.

b) An equation $e \llbracket T \rrbracket$ is redundant in $E_{j}$ if for every ground instance $e \sigma$ of it with $\sigma$ irreducible wrt. $R_{E_{\infty}}$, there exist instances $d_{i}$ in $\operatorname{irred}_{R_{E \infty}}\left(E_{j}\right)$, for $i=1 \ldots m$, such that $e \sigma \succ_{m u l} d_{i}$ and $R_{E_{\infty}} \cup\left\{d_{1}, \ldots, d_{m}\right\} \mid=e \sigma$.

Definition 13: A completion derivation is a sequence of sets of constrained equations $E_{0}, E_{1}, \ldots$ such that $T_{0}$ is true for every equation $e_{0} \llbracket T_{0} \rrbracket$ in $E_{0}$ and

$$
\begin{aligned}
& E_{i}=E_{i-1} \cup\{e \llbracket T \rrbracket\} \quad \text { where } E_{i-1}=e \llbracket T \rrbracket, \text { or } \\
& E_{i}=E_{i-1} \backslash\{e \llbracket T \rrbracket\}
\end{aligned}
$$

Definition 14: Let $E_{0}, E_{1}, \ldots$ be a completion derivation, and let $\pi$ be a basic superposition inference with premises $e_{1} \llbracket T_{1} \rrbracket$ and $e_{2} \llbracket T_{2} \rrbracket$, and with conclusion $e \llbracket T \rrbracket$. Then every inference by basic superposition with premises $e_{1} \sigma$ and $e_{2} \sigma$, and conclusion e $\sigma$ with $T \sigma \equiv$ true, for some ground substitution $\sigma$, is a ground instance $\pi \sigma$ of $\pi$.

The inference $\pi$ is redundant in $E_{j}$ if for every ground instance $\pi \sigma$ of $\pi$ with $\sigma$ irreducible wrt. $R_{E_{\infty}}$, there exist instances $d_{i}$ in $\operatorname{irred}_{R_{E \infty}}\left(E_{j}\right)$, for $i=1 \ldots m$, such that $\max \left(e_{1} \sigma, e_{2} \sigma\right) \succ_{m u l} d_{i}$ and $R_{E_{\infty}} \cup\left\{d_{1} \ldots d_{m}\right\} \vDash e \sigma$, where max denotes maximality wrt. $\succ_{m u l}$.

Definition 15: A completion derivation $E_{0}, E_{1}, \ldots$ is fair if every inference by basic superposition with premises in $E_{\infty}$ is redundant in some $E_{j}$. 
As we can see, in completion derivations we consider instances with substitutions irreducible wrt. $R_{E_{\infty}}$. For example, an equation is redundant if all its instances that are irreducible in that sense can be deduced from other smaller irreducible instances.

However, in practice, during the computation of a fair derivation, one cannot prove the redundancy of equations or inferences in a set $E_{j}$, since at that point $R_{E_{\infty}}$ is unknown. Therefore, sufficient conditions for redundancy have to be used. We will define them in detail at the end of this section, and we suppose for the moment that we can indeed compute fair completion derivations.

Definition 16: Let $E_{0}, E_{1}, \ldots$ be a completion derivation. Then $E_{\infty}$ is complete if every inference by basic superposition with premises in $E_{\infty}$ is redundant in $E_{\infty}$.

Lemma 17: Let $E_{0}, E_{1}, \ldots$ be a completion derivation. Then for every set $E_{j}$ and instance $e \sigma$ in $\operatorname{irred}_{R_{E \infty}}\left(E_{j}\right)$, there are instances $d_{i}$ for $i=1 \ldots m$ in $\operatorname{irred}_{R_{E \infty}}\left(E_{\infty}\right)$, such that $R_{E_{\infty}} \cup\left\{d_{1}, \ldots, d_{m}\right\}=e \sigma$ and $e \sigma \succeq_{m u l} d_{i}$.

Proof. We derive a contradiction from the existence of an instance $e \sigma$ that is minimal (w.r.t. $\succ_{m u l}$ ) in all sets $\operatorname{irred}_{R_{E \infty}}\left(E_{j}\right)$ such that there are no such instances $d_{i}$ in $\operatorname{irred}_{R_{E \infty}}\left(E_{\infty}\right)$. The corresponding equation $e \llbracket T \rrbracket$ in $E_{j}$ is not persistent, because otherwise $e \sigma$ is in irred $R_{E \infty}\left(E_{\infty}\right)$. This means that $e \llbracket T \rrbracket$ is redundant in some $E_{k}$, with $k \geq j$, i.e. there exist instances $d_{q}^{\prime}$ with $q=1 \ldots n$, in $\operatorname{irred}_{R_{E \infty}}\left(E_{k}\right)$ such that $R_{E_{\infty}} \cup\left\{d_{1}^{\prime}, \ldots, d_{n}^{\prime}\right\} \vDash e \sigma$, with $e \sigma \succ_{m u l} d_{q}^{\prime}$. However, if the result holds for the instances $d_{1}^{\prime}, \ldots, d_{n}^{\prime}$ (which must be the case, because $e \sigma$ is minimal), then it also holds for $e \sigma$.

Lemma 18: Let $E_{0}, E_{1}, \ldots$ be a completion derivation. If an inference by basic superposition is redundant in some $E_{j}$, then it also is in $E_{\infty}$.

Proof. Let $\pi$ be an inference with premises $e_{1} \llbracket T_{1} \rrbracket$ and $e_{2} \llbracket T_{2} \rrbracket$, and with conclusion $e \llbracket T \rrbracket$, such that $\pi$ is redundant in $E_{j}$. Then, by definition of redundant inference, for every ground instance $\pi \sigma$ of $\pi$ with $\sigma$ irreducible wrt. $R_{E_{\infty}}$, there exist instances $d_{i}$ in $\operatorname{irred}_{R_{E \infty}}\left(E_{j}\right)$, for $i=1 \ldots m$, such that $\max \left(e_{1} \sigma, e_{2} \sigma\right) \succ_{m u l} d_{i}$ and $R_{E_{\infty}} \cup\left\{d_{1}, \ldots, d_{m}\right\} \vDash e \sigma$. By the previous lemma, each of the instances $d_{i}$ can be deduced from $R_{E_{\infty}}$ and other instances $\left\{d_{1}^{\prime}, \ldots, d_{n}^{\prime}\right\}$ in $\operatorname{irred}_{R_{E \infty}}\left(E_{\infty}\right)$ such that $d_{i} \succeq_{m u l} d_{j}^{\prime}$. This implies that $\pi$ is also redundant in $E_{\infty}$.

Lemma 19: If $E_{0}, E_{1}, \ldots$ is a fair completion derivation, then $E_{\infty}$ is complete.

Proof. By fairness, every inference $\pi$ with premises in $E_{\infty}$ is redundant in some $E_{j}$. By the previous lemma, then $\pi$ is also redundant in $E_{\infty}$, that is, $E_{\infty}$ is complete.

We now apply the same method as above to prove that $E_{\infty}$ is ground confluent. The following lemma states that in fair completion derivations $R_{E_{\infty}}^{*} \equiv \operatorname{irred}_{R_{E \infty}}\left(E_{\infty}\right)$. After this, in lemma 21, we show that $R_{E_{\infty}}^{*} F E_{\infty}$, which, as we know by lemma 9 , implies that $E_{\infty}$ is ground confluent. 
Lemma 20: If $E_{0}, E_{1}, \ldots$ is a fair completion derivation then $R_{E_{\infty}}^{*}=\operatorname{irred}_{R_{E_{\infty}}}\left(E_{\infty}\right)$. Proof. This proof is an easy extension of that of lemma 7, where the same result is proved for sets $E$ that are closed under basic superposition, instead of what we need here: proving it for $E_{\infty}$ which we only know to be complete, i.e. closed up to redundant inferences.

Let $t \sigma \simeq t^{\prime} \sigma$ be a minimal (wrt. $\succ_{m u l}$ ) instance in irred $_{R_{E \infty}}\left(E_{\infty}\right)$ such that $R_{E_{\infty}}^{*} \not=$ $t \sigma \simeq t^{\prime} \sigma$. We will derive a contradiction from the existence of such an equation.

We can suppose w.l.o.g. that $t \sigma \succ t^{\prime} \sigma$. Since $R_{E_{\infty}}^{*} \not t \sigma \simeq t^{\prime} \sigma$, the equation has not generated any rule in $R_{E_{\infty}}$. Therefore $t \sigma$ must be reducible by $R_{E_{\infty}}$, e.g. with a rule $s \theta \Rightarrow s^{\prime} \theta$ generated by an equation $s \theta \simeq s^{\prime} \theta$ smaller than $t \sigma \simeq t^{\prime} \sigma$. Now we have $\left.t \sigma\right|_{u}=s \theta$, where $\left.t\right|_{u}$ cannot be a variable, since $\sigma$ is irreducible, and therefore the following inference can be made:

$$
\frac{s \simeq s^{\prime} \llbracket T^{\prime} \rrbracket \quad t \simeq t^{\prime} \llbracket T \rrbracket}{\left.t\left[s^{\prime}\right]_{u} \simeq t^{\prime} \llbracket T \wedge T^{\prime} \wedge t\right|_{u}=s \rrbracket}
$$

Its conclusion has a ground instance $d$ of the form $t \sigma\left[s^{\prime} \theta\right]_{u} \simeq t^{\prime} \sigma$ such that $R_{E_{\infty}}^{*} \not d$ (otherwise $R_{E_{\infty}}^{*} \models t \sigma \simeq t^{\prime} \sigma$ ). Moreover, $d$ is an instance of this conclusion with a ground substitution that is irreducible by $R_{E_{\infty}}$ (as in lemma 7).

Since $E_{\infty}$ is complete, the inference must be redundant in $E_{\infty}$, i.e. there exist instances $d_{i}$ in irred $_{R_{E \infty}}\left(E_{\infty}\right)$, for $i=1 \ldots m$, such that $t \sigma \simeq t^{\prime} \sigma \succ_{m u l} d_{i}$ and $R_{E_{\infty}} \cup\left\{d_{1}, \ldots, d_{m}\right\}=$ $d$. But if $R_{E_{\infty}}^{*} \not \neq d$ then also $R_{E_{\infty}}^{*} \not \models d_{i}$ for some $d_{i}$, contradicting the minimality of $t \sigma \simeq t^{\prime} \sigma$.

Lemma 21: If $E_{0}, E_{1}, \ldots$ is a fair completion derivation then $R_{E_{\infty}}^{*} \models E_{\infty}$.

Proof. We have $R_{E_{\infty}}^{*}=\operatorname{irred}_{R_{E \infty}}\left(E_{\infty}\right)$ by the previous lemma. Moreover, $R_{E_{\infty}} \cup$ $\operatorname{irred}_{R_{E \infty}}\left(E_{\infty}\right) \models$ irred $_{R_{E \infty}}\left(E_{0}\right)$ is a direct consequence of lemma 17. Now, since $R_{E_{\infty}} \cup$ irred $_{R_{E \infty}}\left(E_{0}\right)=E_{0}$ holds as in lemma 8 (equations in $E_{0}$ have no constraints), and $E_{0}=E_{\infty}$ holds since $E_{i}=E_{i+1}$ for all $i$, together we have $R_{E_{\infty}}^{*}=E_{\infty}$.

Theorem 22: If $E_{0}, E_{1}, \ldots$ is a fair completion derivation then $E_{\infty}$ is ground confluent.

\subsection{Redundancy notions for basic superposition}

In this section we study in which concrete situations the usual notions of redundancy are incorrect when dealing with basic superposition. It is shown that these situations can be avoided by sometimes slightly weakening constraints, in such a way that basic superposition only in the very worst case may degenerate into normal superposition.

Roughly speaking, the notions of redundant axioms and inferences for normal superposition of [BG 91] state that a clause is redundant if all its ground instances can be deduced from smaller instances of other clauses, and an inference is redundant if, for all its instances, the conclusion can be deduced from instances smaller than the maximal premise. These notions include most simplification techniques and critical pair criteria for proving the redundancy of superpositions. For example, the simplification of an 
equation $e$ into $e^{\prime}$ can be modelled in a completion derivation by first adding $e^{\prime}$, and then deleting $e$, which has become redundant.

Below we prove that these notions of redundancy can also be used in basic superposition. However, our notion of redundant equation (defin. 12) requires every instance with an irreducible substitution to be deducible from other smaller instances with irreducible substitutions, and also $R_{E_{\infty}}$ may be used in redundancy proofs:

Example 23: In example 11, the equation $f(g(a)) \simeq b$ is simplified into $f(b) \simeq b$ using $g(x) \simeq b \llbracket x=a \rrbracket$ with the substitution $\sigma$, which is $\{x \mapsto a\}$.

However, $f(g(a)) \simeq b$ does not become redundant by adding $f(b) \simeq b$, because we need $g(x) \simeq b \llbracket x=a \rrbracket$ instantiated with $\sigma$, but $\sigma$ is not irreducible, since $R_{E_{\infty}}$ contains the equation $a \simeq b$, with $a \succ b$.

Before giving other sufficient conditions for redundancy in our framework, let us remark that by our notion of definition 12 we obtain an interesting result: a constrained equation $e \llbracket T \rrbracket$ is redundant (i.e. it can be deleted) if $\sigma$ is the most general unifier of $T$ and, for some variable $x$ in $e, x \sigma$ is reducible by an equation $e^{\prime}$ in some $E_{j}$. This is true because if $x \sigma$ is reducible by $e^{\prime}$ then it is also reducible by some rule in $R_{E_{\infty}}$, and therefore $e \llbracket T \rrbracket$ has no irreducible ground instances at all.

Definition 24: Let $e \llbracket T \rrbracket$ be an equation, and let $\theta$ be the most general unifier of the equality constraint $T$. Then $T$ binds each variable $x$ in $\mathcal{V}$ ars $(e)$ with $x \theta \neq x$ to $x \theta$.

Lemma 25: Let $E_{0}, E_{1}, \ldots$ be a completion derivation. The equation $e \llbracket T \rrbracket$ is redundant in a set $E_{j}$ if

(i) for every ground instance $e \sigma$ there are ground instances $d_{i} \sigma_{i}$ for $i=1 \ldots m$ of equations $d_{i} \llbracket T_{i} \rrbracket$ in $E_{j}$ such that $\left\{d_{1} \sigma_{1}, \ldots, d_{m} \sigma_{m}\right\} \models e \sigma$ and $e \sigma \succ_{m u l} d_{i} \sigma_{i}$, and

(ii) for every $i$ in $1 \ldots m$, and for every $x$ in $\mathcal{V}$ ars $\left(d_{i}\right), T_{i}$ does not bind $x$, or else $x \sigma_{i}=y \sigma$, for some variable $y$ in $e$.

Proof. We have to prove that the conditions imply that for every $e \sigma$ where $\sigma$ is ground and irreducible wrt. $R_{E_{\infty}}$, there exist instances $d_{k}^{\prime}$ in $\operatorname{irred}_{R_{E \infty}}\left(E_{j}\right)$, for $k=1 \ldots n$, such that $e \sigma \succ_{m u l} d_{k}^{\prime}$ and $R_{E_{\infty}} \cup\left\{d_{1}^{\prime}, \ldots, d_{k}^{\prime}\right\}=e \sigma$.

If every substitution $\sigma_{i}$ is irreducible wrt. $R_{E_{\infty}}$, then the result holds. This is certainly the case if for every variable $x$ in every $d_{i}$ we have $x \sigma_{i}=y \sigma$, for some variable $y$ in $e$, since $\sigma$ is irreducible.

Otherwise, if $x \sigma_{i}$ is reducible by $R_{E_{\infty}}$, we can replace $d_{i} \sigma_{i}$ by $d_{i} \theta_{i}$, where $\theta_{i}$ is the ground substitution such that $x \theta_{i}$ is a normal form wrt. $R_{E_{\infty}}$ of $x \sigma_{i}$, and $z \theta_{i}=z \sigma_{i}$ for every other variable $z$ in $d_{i}$. Now $d_{i} \theta_{i}$ is an existing instance of $d_{i}$, since $x$ is not bound by the corresponding constraint $T_{i}$. Moreover, we have $R_{E_{\infty}} \cup\left\{d_{i} \theta_{i}\right\}=d_{i} \sigma_{i}$. By doing so for all such variables $x$, we obtain the instances $d_{k}^{\prime}$ in $\operatorname{irred}_{R_{E \infty}}\left(E_{j}\right)$, for $k=1 \ldots n$, such that $e \sigma \succ_{m u l} d_{k}^{\prime}$ and $R_{E_{\infty}} \cup\left\{d_{1}^{\prime}, \ldots, d_{k}^{\prime}\right\} \models e \sigma$. 
The lemma above means for instance that, roughly speaking, one can apply an equation $e \llbracket T \rrbracket$ in a redundancy proof if, for every variable $x$ in $\mathcal{V} a r s(e), x$ is not bound by $T$, or else the "corresponding" position in the equation proved is also a variable:

Example 26: The equation $h(f(y)) \simeq y \llbracket y=a \rrbracket$ can be simplified* by the equation $f(x) \simeq b \llbracket x=a \rrbracket$ into $h(b) \simeq y \llbracket y=a \rrbracket$, because, although the variable $x$ is bound, its corresponding position in $h(f(y))$ is the variable $y$.

A lemma equivalent to lemma 25 for proving the redundancy of inferences also holds: it is obtained by using the instance of the maximal premise as upper bound for the instances $d_{1}, \ldots, d_{m}$, instead of $e \sigma$.

Might all the conditions of the previous lemma fail, for some variable $x$, then we can always weaken $T$ for $x$ :

Lemma 27: Let $e \llbracket T \rrbracket$ be an equation, and let $\theta$ be the most general unifier of $T$, with $\theta$ of the form $\left\{x_{1} \mapsto t_{1}, \ldots, x_{n} \mapsto t_{n}\right\}$. Now let $\sigma$ be $\left\{x_{1} \mapsto t_{1}\right\}$. Then the equation $e \sigma \llbracket x_{2}=t_{2} \wedge \ldots \wedge x_{n}=t_{n} \rrbracket$, obtained by weakening $e \llbracket T \rrbracket$ for $x_{1}$, is logically equivalent to $e \llbracket T \rrbracket$.

Weakening the constraint of an equation is equivalent to turning basic superposition into normal superposition for the given subterm in the equation $\left(t_{1}\right.$ in the previous lemma), since it becomes again necessary to apply superposition on it, while it was not before weakening.

In fact, one can also apply partial weakening steps, i.e. instantiating the variable $x_{1}$ only with the outermost symbol of the term $t_{1}$ (or doing this several times) if this is enough for fulfilling the conditions of lemma 25. For example, if $t_{1}$ is of the form $f\left(s_{1} \ldots s_{m}\right)$ the constraint becomes $\llbracket T \wedge T^{\prime} \wedge y_{1}=s_{1} \wedge \ldots \wedge y_{m}=s_{m} \rrbracket$ and the substitution $\boldsymbol{\sigma}=\left\{\boldsymbol{x}_{1} \mapsto f\left(y_{1} \ldots y_{m}\right)\right\}$ is applied to the equation, where $y_{1} \ldots y_{m}$ are new variables.

For simplicity, we have not considered here redundancy of equations by subsumption, which can be proved by combining $\succ_{m u l}$ with the subsumption ordering (but note that, in order to fulfil the conditions of lemma 25 , the subsuming constrained equation has to be weakened until its equation part is, in some sense, as instantiated as the subsumed equation).

Practical implementations, such as the one we are working on based on the TRIP system [NOR 90], will show whether it pays off to weaken constraints for simplification steps, or whether it is always more efficient to use basic superposition in its full power. For the moment, it seems to us that some mixed strategy has to be used.

* If we use a notion of simplification where matching has to be compatible with the equality constraints. Here we will not define concrete simplification methods for equality constrained equations. As far as we know, the previous lemma covers all intuitive extensions of known methods to the equality constraint case. 


\section{Completion of first-order clauses by basic superposition}

In this section we extend the techniques defined above to the case of full first-order clauses with equality. As done by Bachmair and Ganzinger in [BG 91], we obtain an unfailing completion procedure for first-order clauses with equality, including powerful notions of redundancy for clauses and inferences. This procedure is refutationally complete and, moreover, very efficient complete strategies can be used for refutational theorem proving with complete sets of clauses.

The main new result given here is that our completion procedure, while conserving these properties, uses an inference system that has as main inference rule the one of strict basic superposition, instead of normal strict superposition, with the corresponding advantages of a more reduced search space and higher termination probabilities.

Moreover, apart from using basic superposition, the new inference system we define below (first proved complete in [Nie 91]) is also interesting because there is only one inference rule for equality factoring, instead of including, apart from "normal" factoring, inference rules for merging paramodulation or equality factoring left and equality factoring right [BG 91]. The fact that we use here this specific inference system does not mean that our methods depend on it: our lifting techniques can be easily adapted to each one of these other systems. Our results can also be extended to calculi which consider only one arbitrary marked negative literal for superposition, as done in [NN 91] for Horn clauses with equality, and in [BG 91] as selection functions on negative literals of full first-order clauses.

In the following ordering $\succ_{C}$ on ground clauses, the terms appearing in antecedents of clauses are slightly more complex than the ones in succedents:

Definition 28: The multiset expression of an equation $t \simeq t^{\prime}$ in a clause $\Gamma \rightarrow \Delta$ is

(i) $\left\{\{t, t\},\left\{t^{\prime}, t^{\prime}\right\}\right\} \quad$ if $t \simeq t^{\prime}$ belongs to $\Gamma$

(ii) $\left\{\{t\},\left\{t^{\prime}\right\}\right\} \quad$ if $t \simeq t^{\prime}$ belongs to $\Delta$

The ordering $\succ_{e}$ on ground equations is defined as the ordering $\succ_{m u l^{2}}$ on their multiset expressions.

The ordering $\succ_{C}$ on ground clauses is defined as the ordering $\succ_{m u} l^{3}$ on the multisets containing the multiset expressions of their equations.

Definition 29: A ground equation $e$ is called maximal (resp. strictly maximal) in a ground clause $C$ if $e \succeq_{e} e^{\prime}$ (resp. $e \succ_{e} e^{\prime}$ ), for every other equation $e^{\prime}$ in $C$.

In the following inference system $\mathcal{B}$ (here $\mathcal{B}$ stands for "Basic superposition") inferences take place only in equations of succedents that are strictly maximal and in equations of antecedents that are maximal, for some ground instance. Moreover, only the maximal terms in each equation are used. These conditions imply that, for each ground inference, the conclusion is strictly smaller (wrt. $\succ_{C}$ ) than the maximal premise. 
Definition 30: The inference rules of $\mathcal{B}$ are the following (we always consider maximality of equations in clauses wrt. $\succ_{e}$ ):

1) strict basic superposition right:

$\frac{\Gamma^{\prime} \rightarrow \Delta^{\prime}, s \simeq s^{\prime} \llbracket T^{\prime} \rrbracket \quad \Gamma \rightarrow \Delta, t \simeq t^{\prime} \llbracket T \rrbracket}{. \Gamma^{\prime}, \Gamma \rightarrow \Delta^{\prime}, \Delta,\left.t\left[s^{\prime}\right]_{u} \simeq t^{\prime} \llbracket T \wedge T^{\prime} \wedge t\right|_{u}=s \rrbracket} \quad$ where $\left.t\right|_{u} \notin \mathcal{V}$ ars $(t)$

if $\left.\llbracket T \wedge T^{\prime} \wedge t\right|_{u}=s \rrbracket \sigma$ is true for some ground substitution $\sigma$ such that

a) $t \sigma \succ t^{\prime} \sigma, s \sigma \succ s^{\prime} \sigma$, and $t \sigma \simeq t^{\prime} \sigma \succ_{e} s \sigma \simeq s^{\prime} \sigma$

b) $s \sigma \simeq s^{\prime} \sigma$ is strictly maximal in $\Gamma^{\prime} \sigma \rightarrow \Delta^{\prime} \sigma, s \sigma \simeq s^{\prime} \sigma$

c) $t \sigma \simeq t^{\prime} \sigma$ is strictly maximal in $\Gamma \sigma \rightarrow \Delta \sigma, t \sigma \simeq t^{\prime} \sigma$.

2) strict basic superposition left:

$$
\frac{\Gamma^{\prime} \rightarrow \Delta^{\prime}, s \simeq s^{\prime} \llbracket T^{\prime} \rrbracket \quad \Gamma, t \simeq t^{\prime} \rightarrow \Delta \llbracket T \rrbracket}{\Gamma^{\prime}, \Gamma, t\left[s^{\prime}\right]_{u} \simeq t^{\prime} \rightarrow \Delta^{\prime},\left.\Delta \llbracket T \wedge T^{\prime} \wedge t\right|_{u}=s \rrbracket} \quad \text { where }\left.t\right|_{u} \notin \mathcal{V} \operatorname{ars}(t)
$$

if $\left.\llbracket T \wedge T^{\prime} \wedge t\right|_{u}=s \rrbracket \sigma$ is true for some ground substitution $\sigma$ such that

a) $t \sigma \succ t^{\prime} \sigma$ and $s \sigma \succ s^{\prime} \sigma$

b) $s \sigma \simeq s^{\prime} \sigma$ is strictly maximal in $\Gamma^{\prime} \sigma \rightarrow \Delta^{\prime} \sigma, s \sigma \simeq s^{\prime} \sigma$

c) $t \sigma \simeq t^{\prime} \sigma$ is maximal in $\Gamma \sigma, t \sigma \simeq t^{\prime} \sigma \rightarrow \Delta \sigma$.

3) equality resolution:

$$
\frac{\Gamma, t \simeq t^{\prime} \rightarrow \Delta \llbracket T \rrbracket}{\Gamma \rightarrow \Delta \llbracket T \wedge t=t^{\prime} \rrbracket}
$$

if $\llbracket T \wedge t=t^{\prime} \rrbracket \sigma$ is true for some ground substitution $\sigma$ such that

a) $t \sigma \simeq t^{\prime} \sigma$ is maximal in $\Gamma \sigma, t \sigma \simeq t^{\prime} \sigma \rightarrow \Delta \sigma$.

4) factoring:

$$
\frac{\Gamma \rightarrow \Delta, t \simeq s, t^{\prime} \simeq s^{\prime} \llbracket T \rrbracket}{\Gamma, s \simeq s^{\prime} \rightarrow \Delta, t \simeq s \llbracket T \wedge t=t^{\prime} \rrbracket}
$$

if $\llbracket T \wedge t=t^{\prime} \rrbracket \sigma$ is true for some ground substitution $\sigma$ such that

a) $t \sigma \succ s \sigma$ and $t^{\prime} \sigma \succ s^{\prime} \sigma$

b) $t \sigma \simeq s \sigma$ is maximal in $\Gamma \sigma \rightarrow \Delta \sigma, t \sigma \simeq s \sigma, t^{\prime} \sigma \simeq s^{\prime} \sigma$.

Note that our inference rule for factoring is a generalization to the equality case of "normal" factoring. For instance, if $t$ and $t^{\prime}$ are atoms, then both $s$ and $s^{\prime}$ are the symbol true and the equation true $\simeq$ true can be omitted in the antecedent. 
In order to prove the correctness of completion procedures based on this inference system $\mathcal{B}$, we will proceed in a similar way as done in the previous section for the equational case. In fact, we will extend almost all the definitions and results to the case of first-order clauses with equality, of which equations are a proper subset. For instance, definitions 31 - 36 are extensions of the equivalent ones in the previous section, and the same thing happens with the lemmas $37-39$ and 41 , whose proofs are omitted here.

Now first we associate to a set of constrained clauses $S$ a canonical set of ground rewrite rules $R_{S}$, in a similar way as it was done for the equational case. After this, it will be shown that, in a fair completion derivation for first-order clauses $S_{0}, S_{1}, \ldots$, if the empty clause* is not in $S_{\infty}$, then $R_{S_{\infty}}^{*}=S_{\infty}$, i.e. $S_{\infty}$ has a model. So we obtain the result (just as $R_{E_{\infty}}^{*} \models E_{\infty}$ implied the confluence of $E_{\infty}$ ) that the completion procedure is refutationally complete.

Definition 31: Let $C$ be a ground instance $\Gamma \rightarrow \Delta, t \simeq s$ of a clause $D \llbracket T \rrbracket$ in a set $S$, i.e. $C$ is $D \sigma$ for some ground substitution $\sigma$ such that $T \sigma \equiv$ true.

Then $C$ generates a rule $t \Rightarrow s$ if the following conditions hold:

(1) $R_{C}^{*} \not \neq C$

(2) $t \simeq s$ is maximal (wrt. $\succ_{e}$ ) in $C$ with $t \succ s$

(3) $R_{C}^{*} \not \models s \simeq s^{\prime}$, for every $t \simeq s^{\prime}$ in $\Delta$

(4) $t$ is irreducible by $R_{C}$

(5) $\sigma$ is irreducible by $R_{C}$

where $R_{C}$ is the set of rules generated by ground instances smaller than $C$ (wrt. $\succ_{C}$ ) of clauses in $S$.

The set of rules generated by all ground instances of clauses in $S$ is denoted by $R_{S}$.

Definition 32: Let $S_{0}, S_{1}, \ldots$ be a sequence of sets of constrained clauses.

a) The set $S_{\infty}$ of persistent clauses in $S_{0}, S_{1}, \ldots$ is defined as $\cup_{j}\left(\cap_{k \geq j} S_{k}\right)$.

b) A clause $C \llbracket T \rrbracket$ is redundant in $S_{j}$ if for every ground instance $C \sigma$ of it with $\sigma$ irreducible wrt. $R_{S_{\infty}}$, there exist instances $D_{i}$ in $\operatorname{irred}_{R_{S \infty}}\left(S_{j}\right)$, for $i=1 \ldots m$, such that $C \sigma \succ_{C} D_{i}$ and $R_{S_{\infty}} \cup\left\{D_{1}, \ldots, D_{m}\right\} \models C \sigma$.

Definition 33: A theorem proving derivation is a sequence of sets of constrained clauses $S_{0}, S_{1}, \ldots$ such that $T_{0}$ is true for every clause $C_{0} \llbracket T_{0} \rrbracket$ in $S_{0}$ and

$$
\begin{array}{ll}
S_{i}=S_{i-1} \cup\{C \llbracket T \rrbracket\} & \text { where } S_{i-1} \vdash C \llbracket T \rrbracket, \text { or } \\
S_{i}=S_{i-1} \backslash\{C \llbracket T \rrbracket\} & \text { if } C \llbracket T \rrbracket \text { is redundant in } S_{i-1}
\end{array}
$$

* The empty clause with a satisfiable constraint. Clauses with unsatisfiable constraints are tautologies. 
Definition 34: Let $S_{0}, S_{1}, \ldots$ be a theorem proving derivation, and let $\pi$ be an inference of $\mathcal{B}$ with premises $C_{1} \llbracket T_{1} \rrbracket, \ldots, C_{n} \llbracket T_{n} \rrbracket$, and with conclusion $C \llbracket T \rrbracket$.

Then every existing inference of $\mathcal{B}$ with premises $C_{1} \sigma, \ldots, C_{n} \sigma$, and conclusion $C \sigma$ with $T \sigma \equiv$ true, for some ground substitution $\sigma$, is a ground instance $\pi \sigma$ of $\pi$.

The inference $\pi$ is redundant in $S_{j}$ if for every ground instance $\pi \sigma$ of $\pi$ with $\sigma$ irreducible wrt. $R_{S_{\infty}}$, there exist instances $D_{i}$ in $\operatorname{irred}_{R_{S \infty}}\left(S_{j}\right)$, for $i=1 \ldots m$, such that $\max \left(C_{1} \sigma, \ldots, C_{n} \sigma\right) \succ_{C} D_{i}$ and $R_{S_{\infty}} \cup\left\{D_{1}, \ldots, D_{m}\right\} \mid=C \sigma$, where max denotes maximality wrt. $\succ_{C}$.

Definition 35: A theorem proving derivation $S_{0}, S_{1}, \ldots$ is fair if every inference of the inference system $\mathcal{B}$ with premises in $S_{\infty}$ is redundant in some $S_{j}$.

Definition 36: Let $S_{0}, S_{1}, \ldots$ be a theorem proving derivation. Then $S_{\infty}$ is complete if every inference of the inference system $\mathcal{B}$ with premises in $S_{\infty}$ is redundant in $S_{\infty}$.

Lemma 37: Let $S_{0}, S_{1}, \ldots$ be a theorem proving derivation. Then for every set $S_{j}$ and instance $C$ in irred $_{R_{S \infty}}\left(S_{j}\right)$, there are instances $D_{i}$ for $i=1 \ldots m$ in irred $_{R_{S \infty}}\left(S_{\infty}\right)$, such that $R_{S_{\infty}} \cup\left\{D_{1}, \ldots, D_{m}\right\}=C$ and $C \succeq_{C} D_{i}$.

Lemma 38: Let $S_{0}, S_{1}, \ldots$ be a theorem proving derivation. If an inference is redundant in some $S_{j}$, then it also is in $S_{\infty}$.

Lemma 39: If $S_{0}, S_{1}, \ldots$ is a fair theorem proving derivation, then $S_{\infty}$ is complete.

For technical reasons which we explain in the lemma below, we apply a minimal weakening step to consequences of inferences with non-horn clauses where the left premise is of the form $\Gamma \rightarrow \Delta, x \simeq s, x \simeq s^{\prime} \llbracket T \rrbracket$ and where $x \simeq s$ is the equation superposed on the right premise using $x$ as left hand side. In fact, this weakening is not really needed*, but without doing it all proof techniques become quite more complicated and a lot of power wrt. redundancy (e.g. lemma 43) is then lost, which we think does not pay off. Note that this quite special case only applies to non-Horn clauses, since there are at least two equations in the succedent, and only if $x$ does not appear in $\Gamma$ (otherwise $x \sigma \simeq s \sigma$ cannot be maximal for any ground substitution $\sigma$ ). Now after an inference step by e.g. basic superposition left

$$
\frac{\Gamma \rightarrow \Delta, x \simeq s, x \simeq s^{\prime} \llbracket T \rrbracket \quad \Gamma^{\prime}, t \simeq t^{\prime} \rightarrow \Delta^{\prime} \llbracket T^{\prime} \rrbracket}{\Gamma, \Gamma^{\prime}, t[s]_{u} \simeq t^{\prime} \rightarrow \Delta, \Delta^{\prime},\left.x \simeq s^{\prime} \llbracket T \wedge T^{\prime} \wedge t\right|_{u}=x \rrbracket} \quad \text { where }\left.t\right|_{u} \notin \mathcal{V a r s}(t)
$$

the conclusion is minimally weakened for the variable $x$ (as done in section 3.2 ), i.e. the variable $x$ in the clause is instantiated with the outermost symbol of the term $\left.t\right|_{u}$ it is superposed upon.

More precisely, if $\left.t\right|_{u}$ is of the form $f\left(t_{1} \ldots t_{n}\right)$ the constraint of the conclusion becomes $\llbracket T \wedge T^{\prime} \wedge x_{1}=t_{1} \wedge \ldots \wedge x_{n}=t_{n} \rrbracket$ and the substitution $\sigma=\left\{x \mapsto f\left(x_{1} \ldots x_{n}\right)\right\}$ is applied to the whole constrained clause. From now on we suppose that this weakening is done after all such inferences by strict superposition left and right.

* This has been recently pointed out to us by H. Ganzinger. 
The only lemma of this section that is significantly different to the equational case is the following one. The reason is that it depends on the inference system used.

Lemma 40: Let $S_{0}, S_{1}, \ldots$ be a fair theorem proving derivation, such that $S_{\infty}$ does not contain the empty clause. Then $R_{S_{\infty}}^{*}=\operatorname{irred}_{R_{S \infty}}\left(S_{\infty}\right)$.

Proof. Let $C \sigma$ be a minimal (wrt. $\succ_{C}$ ) instance irred $_{R_{S_{\infty}}}\left(S_{\infty}\right)$ of a clause $C \llbracket T \rrbracket$ in $S_{\infty}$, such that $R_{S_{\infty}}^{*} \not \models C \sigma$. We will derive a contradiction from the existence of such a clause. There are several cases to be analyzed, depending on which one is the maximal equation in $C \sigma$ :

a) Let $C \sigma$ be a clause $\Gamma \sigma \rightarrow \Delta \sigma, t \sigma \simeq t^{\prime} \sigma$, with a maximal equation $t \sigma \simeq t^{\prime} \sigma$, and $t \sigma \succ t^{\prime} \sigma$. Since $R_{S_{\infty}}^{*} \not \models C \sigma$, the clause $C \sigma$ has not generated the rule $t \sigma \Rightarrow t^{\prime} \sigma$. This must be because one of the conditions 3 ) or 4) of definition 31 do not hold.

a1) If condition 3) does not hold, then $\Delta \sigma$ must be of the form $\Delta^{\prime} \sigma, s \sigma \simeq s^{\prime} \sigma$, where $t \sigma$ is $s \sigma$ and $R_{C \sigma}^{*}=t^{\prime} \sigma \simeq s^{\prime} \sigma$. In this case, consider the following inference $\pi$ by factoring

$$
\frac{\Gamma \rightarrow \Delta^{\prime}, t \simeq t^{\prime}, s \simeq s^{4} \llbracket T \rrbracket}{\Gamma, t^{\prime} \simeq s^{\prime} \rightarrow \Delta^{\prime}, t \simeq t^{\prime} \llbracket T \wedge t=s \rrbracket}
$$

Its conclusion has a ground instance $D$ of the form $\Gamma \sigma, t^{\prime} \sigma \simeq s^{\prime} \sigma \rightarrow \Delta^{\prime} \sigma, t \sigma \simeq t^{\prime} \sigma$ such that $R_{S_{\infty}}^{*} \not \models D$. Moreover, $D$ is an instance of this conclusion with a ground substitution that is irreducible by $R_{S_{\infty}}$.

Since $S_{\infty}$ is complete, $\pi$ must be redundant in $S_{\infty}$. But then there exist instances $D_{1}, \ldots, D_{m}$ in $\operatorname{irred}_{R_{S_{\infty}}}\left(S_{\infty}\right)$ such that $R_{S_{\infty}} \cup\left\{D_{1}, \ldots, D_{m}\right\} \mid=D$ and $C \sigma \succ_{C} D_{i}$. Now $R_{S_{\infty}}^{*} \not \models D$ implies that $R_{S_{\infty}}^{*} \not \models D_{i}$ for at least one $D_{i}$, which contradicts the minimality of $\stackrel{C}{\sigma} \sigma$.

a2) If condition 4) does not hold, then $t \sigma$ is reducible by $R_{C \sigma}$, e.g. with a rule $s \theta \Rightarrow s^{\prime} \theta$ generated by a clause $C^{\prime} \theta$ smaller than $C \sigma$. Let $C^{\prime}$ be a clause $\Gamma^{\prime} \rightarrow \Delta^{\prime}, s \simeq s^{\prime}$ in $S_{\infty}$ and $\left.t \sigma\right|_{u}=s \theta$. Now consider the inference $\pi$ by strict superposition right

$$
\frac{\Gamma^{\prime} \rightarrow \Delta^{\prime}, s \simeq s^{\prime} \llbracket T^{\prime} \rrbracket \quad \Gamma \rightarrow \Delta, t \simeq t^{\prime} \llbracket T \rrbracket}{\Gamma^{\prime}, \Gamma \rightarrow \Delta^{\prime}, \Delta,\left.t\left[s^{\prime}\right]_{u} \simeq t^{\prime} \llbracket T \wedge T^{\prime} \wedge t\right|_{u}=s \rrbracket}
$$

Its conclusion has a ground instance $D$ of the form $\Gamma^{\prime} \theta, \Gamma \sigma \rightarrow \Delta^{\prime} \theta, \Delta \sigma, t \sigma\left[s^{\prime} \theta\right]_{u} \simeq t^{\prime} \sigma$, such that $R_{S_{\infty}}^{*} \not \models D$. Moreover, $D$ is an instance of this conclusion with a ground substitution that is irreducible by $R_{S_{\infty}}$. This is true since $\sigma$ is irreducible wrt. $R_{S_{\infty}}$, $\theta$ is irreducible wrt. $R_{C^{\prime} \theta}$, and since we apply weakening steps for certain non-Horn clauses, as defined above. Since $S_{\infty}$ is complete, $\pi$ must again be redundant in $S_{\infty}$, which, as above, leads to a contradiction with the minimality of $C \sigma$.

b) If $C \sigma$ is a clause $\Gamma \sigma, t \sigma \simeq t^{\prime} \sigma \rightarrow \Delta \sigma$, where $t \sigma \simeq t^{\prime} \sigma$ is maximal in $C \sigma$, and $t \sigma$ is $t^{\prime} \sigma$, then consider the following equality resolution inference:

$$
\frac{\Gamma, t \simeq t^{\prime} \rightarrow \Delta \llbracket T \rrbracket}{\Gamma \rightarrow \Delta \llbracket T \wedge t=t^{\prime} \rrbracket}
$$


The conclusion of this inference has a ground instance $D$ of the form $\Gamma \sigma \rightarrow \Delta \sigma$, such that $R_{S_{\infty}}^{*} \not \models D$. Since the inference is redundant, as above, a contradiction is obtained.

c) The only remaining case is that $C \sigma$ is a clause $\Gamma \sigma, t \sigma \simeq t^{\prime} \sigma \rightarrow \Delta \sigma$, where $t \sigma \simeq t^{\prime} \sigma$ is maximal in $C \sigma$ and $t \sigma \succ t^{\prime} \sigma$. In this case $R_{S_{\infty}}^{*} \models t \sigma \simeq t^{\prime} \sigma$, because $R_{S_{\infty}}^{*} \not \models C \sigma$. Then $t \sigma$ must be reducible by a rule $s \theta \Rightarrow s^{\prime} \theta$ in $R_{S_{\infty}}$ generated by a clause in $S_{\infty}$ of the form $\Gamma^{\prime} \rightarrow \Delta^{\prime}, s \simeq s^{\prime} \llbracket T^{\prime} \rrbracket$, where $\left.t \sigma\right|_{u}=s \theta$. The following inference $\pi$ by strict basic superposition left can then be made:

$$
\frac{\Gamma^{\prime} \rightarrow \Delta^{\prime}, s \simeq s^{\prime} \llbracket T^{\prime} \rrbracket \quad \Gamma, t \simeq t^{\prime} \rightarrow \Delta \llbracket T \rrbracket}{\Gamma^{\prime}, \Gamma, t\left[s^{\prime}\right]_{u} \simeq t^{\prime} \rightarrow \Delta^{\prime},\left.\Delta \llbracket T \wedge T^{\prime} \wedge t\right|_{u}=s \rrbracket}
$$

For the corresponding ground instance, $C \sigma$ is the maximal premise, and, as in case a2), for its conclusion $D$ we have $R_{S_{\infty}}^{*} \not \models D$. This implies as before that, since $\pi$ is redundant, a contradiction is obtained.

Lemma 41: Let $S_{0}, S_{1}, \ldots$ be a fair theorem proving derivation. Then $R_{S_{\infty}}^{*} \models S_{\infty}$.

Theorem 42: Let $S_{0}, S_{1}, \ldots$ be a fair theorem proving derivation. Then $S_{0}$ is inconsistent if, and only if, the empty clause belongs to some $S_{j}$.

Proof. If the empty clause belongs to some $S_{j}$, then, since $S_{i}$ is logically equivalent to $S_{i+1}$ for all $i, S_{0}$ is inconsistent. For the reverse implication, suppose the empty clause belongs to no $S_{j}$. Then it is not in $S_{\infty}$, and by the previous lemma, $R_{S_{\infty}}^{*} \models S_{\infty}$. But then $S_{0}$ must be consistent, since it also has the model $R_{S_{\infty}}^{*}$.

With respect to the redundancy notions, again the same discussion as in the previous section applies. All known redundancy notions can be applied, although sometimes weakening is needed. Therefore completion based on the inference rule of basic superposition strictly improves normal superposition-based completion. The following lemma, equivalent to lemma 25 , tells us when constraint weakening has to be applied in redundancy proofs for first-order clauses:

Lemma 43: Let $S_{0}, S_{1}, \ldots$ be a theorem proving derivation. The clause $C \llbracket T \rrbracket$ is redundant in a set $S_{j}$ if

(i) for every ground instance $C \sigma$, there are ground instances $D_{i} \sigma_{i}$ for $i=1 \ldots m$ of clauses $D_{i} \llbracket T_{i} \rrbracket$ in $S_{j}$ such that $\left\{D_{1} \sigma_{1}, \ldots, D_{m} \sigma_{m}\right\}=C \sigma$ and $C \sigma \succ_{C} D_{i} \sigma_{i}$, and moreover

(ii) for every $i$ in $1 \ldots m$, and for every $x$ in $\mathcal{V}$ ars $\left(D_{i}\right), T_{i}$ does not bind $x$, or else $x \sigma_{i}=y \sigma$, for some variable $y$ in $C$.

The interest of applying basic superposition to completion of first-order clauses with equality lies not only in the gain of efficiency as a consequence of the more reduced search space, but also in the higher probability of obtaining complete systems. By using such complete systems $S$, i.e. sets of clauses in which no more non-redundant inferences can be computed, very efficient complete strategies can be applied for refutational theorem proving, since no new inferences between clauses in $S$ have to be computed. 


\section{Further work}

Some of the techniques of this paper can be applied to other kinds of constraints. Here we briefly outline some results of our follow-up paper [NR 91] on the combination of basic superposition modelled by the use of equality constraints, and the notion of ordering constraints. The interest of similar ordering constraints has been pointed out earlier, e.g. in [KKR 90], but, as far as we know, no completeness proofs had been found up to now.

The basic idea is very simple. In ordered inference rules like superposition the search space is reduced by selecting only the maximal terms in the maximal literals to paramodulate upon. Therefore, if a clause is obtained in an inference, we are in fact only interested in those ground instances of it for which the literal (and term) selected is really the biggest one. This information can be kept in its constraint. Future choices of maximal literals that are incompatible with this constraint can then be shown to be unnecessary by proving the unsatisfiability of constraints (the satisfiability of ordering constraints is shown to be decidable in (Com 90]).

For example, if we denote by $t \simeq t^{\prime} \llbracket T \rrbracket$ the ground instances of an equation $t \simeq t^{\prime}$ satisfying the combined ordering and equality constraint $T$, then the inference rule of basic superposition with ordering constraints for the equational case is:

$$
\frac{s \simeq s^{\prime} \llbracket T^{\prime} \rrbracket \quad t \simeq t^{\prime} \llbracket T \rrbracket}{\left.t\left[s^{\prime}\right]_{u} \simeq t^{\prime} \llbracket T^{\prime} \wedge T \wedge s \succ s^{\prime} \wedge t \succ t^{\prime} \wedge t\right|_{u}=s \rrbracket} \quad \text { where }\left.t\right|_{u} \notin \mathcal{V} \operatorname{ars}(t)
$$

which, as we can see, is a very powerful and also elegant representation for ordered inference rules, since information from the meta-level, such as the ordering restrictions and accumulated unifiers generated in ancestors, is included into the formulae and used later on.

Especially in the case of full first-order clauses, but also in the equational case, the ordering constraints become quickly very restrictive, which cuts down the search space drastically. We have reasons to believe that complete systems can be obtained in many more cases, including full first-order specifications. In [NR 91] we define a completion procedure for full first-order clauses with ordering constraints where, as above, redundant inferences can be ignored and redundant clauses can be deleted without loosing completeness. This improves the techniques for ordering constrained completion for the equational case given in [Pet 90], since we can deal with full first-order clauses, we do not need to compute additional kinds of inferences, we allow initial axioms with constraints, and we can combine our methods with basic superposition. In [NR 91] we also report two new results needed for efficiently dealing with ordering constraints.

Acknowledgements: We wish to thank Marianne Haberstrau, Fernando Orejas, Pilar Nivela, Harald Ganzinger and Leo Bachmair and all those who commented our preliminary manuscript introducing this kind of techniques during the RTA '91 conference. 


\section{References}

[BDP 89] L. Bachmair, N. Dershowitz, D. Plaisted: Completion without failure. In H. AitKaci and M. Nivat, editors, Resolution of equations in algebraic structures, vol 2: Rewriting Techniques, pp 1-30, Academic Press, (1989).

[BG 91] L. Bachmair, H. Ganzinger: Completion of first-order clauses with equality. (final version) 2nd Intl. Workshop on Conditional and Typed Term Rewriting, Montreal, LNCS 516, pp. 162-181, (1991).

[Com 90] H. Comon: Solving Symbolic Ordering Constraints. In proc. 5th IEEE Symp. Logic in Comp. Sc. Philadelphia. (June 1990).

[DJ 90] N. Dershowitz, J-P. Jouannaud: Rewrite systems, in Handbook of Theoretical Computer Science, vol. B: Formal Methods and Semantics. (J. van Leeuwen, ed.), North Hollad, Amsterdam, 1990.

[DJ 91] N. Dershowitz, J-P. Jouannaud: Notations for Rewriting. in Bulletin of the EATCS, no. 43, Feb 1991.

[HR 89] J. Hsiang, M. Rusinowitch: Proving refutational completeness of theorem proving strategies: The transfinite semantic tree method. Submitted for publication (1989).

[Hul 80] J.M. Hullot: Compilation de Formes Canoniques dans les Teories Equationnelles, These de 3eme Cycle, Universite de Paris Sud, 1980.

[KB 70] D.E. Knuth, P.B. Bendix: Simple word problems in universal algebras. J. Leech, editor, Computational Problems in Abstract Algebra, 263-297, Pergamon Press, Oxford, 1970.

[KKR 90] C. and H. Kirchner, M. Rusinowitch: Deduction with Symbolic Constraints. Revue Francaise d'Intelligence Artificielle. Vol 4. No. 3. pp. 9-52. Special issue on automatic deduction. (1990).

[Nie 91] R. Nieuwenhuis: First-order completion techniques. Research report UPC-LSI, 1991.

[NN 91] R. Nieuwenhuis, P. Nivela: Efficient deduction in equality Horn logic by Horncompletion, Information Processing Letters, no. 39, pp. 1-6, July 1991.

[NO 91] R. Nieuwenhuis, F. Orejas: Clausal Rewriting. 2nd Intl. Workshop on Conditional and Typed Term Rewriting, Montreal, LNCS 516, pp. 246-261, (1991).

[NOR 90] R. Nieuwenhuis, F. Orejas, A. Rubio: TRIP: an implementation of clausal rewriting. In Proc. 10th Int. Conf. on Automated Deduction. Kaiserslautern, 1990. LNCS, pp 667-668.

[NR 91] R. Nieuwenhuis, A. Rubio: Theorem Proving with Ordering Constrained Clauses. Research report UPC-LSI, 1991. (submitted).

[Pet 90] G.E. Peterson: Complete Sets of Reductions with Constraints. In Proc. 10th Int. Conf. on Automated Deduction. Kaiserslautern, 1990. LNCS, pp 381-395.

[Rus 87] M. Rusinowitch: Theorem-proving with resolution and superposition: an extension of Knuth and Bendix procedure as a complete set of inference rules. Report 87-R128, CRIN, Nancy, 1987. 